\title{
A Survey of Software Quality Assurance and Testing Practices and Challenges in Bangladesh
}

\author{
S. M. Abdur Rouf Bhuiyan \\ Department of Computer Science \\ American International University-Bangladesh \\ Dhaka, Bangladesh \\ A. Z. M. Ehtesham Chowdhury \\ Department of Computer Science \\ American International University-Bangladesh \\ Dhaka, Bangladesh
}

\author{
Md Shamsur Rahim \\ Department of Computer Science \\ American International University-Bangladesh \\ Dhaka, Bangladesh \\ Md Hasibul Hasan \\ Department of Computer Science \\ American International University-Bangladesh \\ Dhaka, Bangladesh
}

\begin{abstract}
Software Quality Assurance and Testing (SQAT) is an integral part of software development. Without the SQAT activities, a software may lead to catastrophic consequences. However, there are many challenges in performing SQAT activities. The goal of this survey was to identify the challenges along with the practices of SQAT activities in Bangladesh. The survey was developed using qualitative research methodologies. The survey was conducted between August, 2017 and January, 2018. In total, 47 organizations participated in the survey. Majority of the software firms that participated in the survey were from Dhaka, the hub of IT sector in Bangladesh. The survey was designed based on four main objectives namely characterizing the organizations, identifying SQAT practices, capturing the essence of testing team along with testing tools and understanding the SQAT challenges, training, education as well as career prospect. This paper presents the findings of the survey and provides recommendations and future research directions for academia as well as for the industry.
\end{abstract}

\section{General Terms}

Computer Science, Software Quality Assurance, Software Testing, Software Engineering

\section{Keywords}

Software Quality Assurance, Software Testing, Software Quality Assurance and Testing, Survey, Software Quality Assurance and Testing Practices, Bangladesh

\section{INTRODUCTION}

Software Quality Assurance (SQA) is an umbrella activity that can be defined as a systematic approach to evaluating the quality of a software and is associated with software product standard, process and procedures. Software quality has a profound impact on our daily lives, e.g. economy, health, safety, security. In many respects, SQA plays a vital role in software development. On the other hand,
Software Testing (ST) is the most important part of SQA and the most commonly performed SQA activity. It is a product-oriented activity where the basic idea is to execute a software and observe its behavior or outcomes. Although, Software Quality Assurance and Testing (SQAT) is a costly activity in software development, still it is an integral part and highly important in software development. The absence or insufficient SQAT activities may lead to economic damages as well as catastrophic consequences. For instances, according to a report [18] by the American National Institute of Standards and Technology (NIST), the United States faces the negative impact of $\$ 62$ billion USD per year due to the lack of SQAT infrastructure. Undoubtedly, the challenges are similar in other counties. For the emerging leaders like Bangladesh in the Information Technology (IT) sector, it is highly important to identify these challenges first, in order to overcome. Although, several surveys have been conducted to identify the practices and challenges of software testing in several countries like Australia [11], Canada, USA, India, Sweden, Finland [6] over the years, however, no such survey on software testing in Bangladesh has ever been conducted to the best of authors knowledge. A survey of software quality assurance and testing practices and challenges in Bangladesh is highly demandable to the practitioners, researchers and policy makers. Because, Bangladesh has been witnessing tremendous growth in the software development organizations in the past 15 years. Now-adays Bangladeshi IT firms are developing software systems that are used not only in Bangladesh but also in many countries across the world. It is expected that IT sector would add 7.28 percent to the GDP growth of Bangladesh by the end of 2021 [1]. The authors along with the assistance of current students and alumni of American International University-Bangladesh (AIUB) conducted a survey on software quality assurance and testing practices in Bangladesh between August, 2017 and January, 2018. A wide variety of IT firms, from CMMI Level 5 to newly established, from domestic to foreign-owned, were invited to participate in this survey. There are several objectives behind conducting this survey: Firstly, the broad objective of this survey is to identify the current software testing practices, trends and challenges in Bangladesh. Secondly, to identify the testing methodologies and other QA prac- 
tices. Thirdly, to identify the programmer/tester ratio and job satisfaction of the test engineers in terms of role and salary. Fourthly, to understand the role and importance of the formal testing team in software development organizations. Fifthly, to pinpoint the importance of automated testing and categories of tools used in software testing. Sixthly, to identify the career prospect of Bangladeshi test engineers. Seventhly, to identify the process maturity level of the IT firms and to identify the key areas that are required to improve. Eighthly, to identify the background knowledge and skills of test engineers. Ninthly, to identify the percentage of effort spent in software testing in a software development project on an average. Finally, to provide guidance for updating software testing curriculum in the universities and training centers. This paper reported the observations obtained from 47 respondents who successfully completed all the questionnaire. The authors believe that in terms of Bangladeshi Software Industry, the sample is enough to obtain interesting findings similar to previous related studies [15] [5]. Most of the software firms participated in this survey are from Dhaka, the capital of Bangladesh. It is because, Dhaka is presently the hub of IT industry in Bangladesh. The rest of the paper is structured as follows: section 2 discusses the previous works related to the paper, section 3 describes the objective of the survey, survey and sample selection method. Section 4 presents the results obtained from the survey. Section 5 discusses the findings of the survey and provides recommendations based on the findings and section 6 concludes the paper and provides future research directions.

\section{RELATED WORKS}

Due to the importance of SQAT process of developing quality software, several types of research have conducted around the world to identify the practices and challenges. The first research has been directed back to 1983 in the USA which was focused on test procedures [7]. Later, almost all the researches conducted regarding this interest were country specific. For instance, a survey was conducted on software testing practices in Australia where 65 organizations responded [11]. The goal of this survey was to report the current testing practices in Australia as well as their recommendations and observations. Similar researches have been steered in Canada, Finland, Sweden, UK [6]. With the growth of ICT sector, software engineering researches in Bangladesh also has gained attentions. Over the years, several types of research have been performed on the different aspects of software engineering [14] [13] [3] [12] [16] [2]. However, to the best of authors knowledge no research has been executed on software testing practices in Bangladesh. Due to the expanding growth and importance of Bangladeshi ICT sector, the authors believe that it is essential to carry out a survey on software testing practices and challenges in Bangladesh. 3.

\section{RESEARCH METHODOLOGY}

This research's tactic is directed and developed using the qualitative research methodologies. The qualitative research method is popular to derive and explore the social, cultural and economic aspects of communities [9]. The qualitative research approaches play an important role to comprehend the insights of a field and the diversities among the contents of that field [17]. This method also helps to dig a context in depth to acquire the vital information [8]. This research is a survey-oriented study which includes the face-to-face interviews where participants answer a sequence of questions in different segments. In following subsections, the detailed description of the survey is deliberated.

\subsection{Survey Objective}

The survey was developed in such a way so that it could accomplish the first five objectives defined in the introduction area. Other five objectives are considered as the consequence of the stream of preceding objectives. The most important objective of this survey is to determine the software testing practices in Bangladeshi IT industries which incorporate the software testing methodologies, criteria for selecting appropriate method for testing from diverse testing methods, understanding the popularity of techniques and tools for testing from diverse software development industries and the relations among the development and testing teams. The subordinate objective of this survey was to deliver understandings from diversified ICT industries of Bangladesh. It would aid the training centers and educational institutes to dynamically modify their course contents and curriculum regarding software testing as the industry demands. This survey possibly will help the educational institutes in scheming the courses on software quality assurance for Bangladesh. Additionally, this survey can aid to offer future research direction in the software quality assurance field, more to say in the software engineering field. The questionnaires of the survey were segmented and defined based on hypotheses derived from the above discussed objectives.

\subsection{Survey Description}

The participants of the survey were the junior to senior personnel of different software firms who were directly or indirectly involved with software quality assurance and testing practices. In this survey, four major areas are explored to encounter the objectives defined in the previous section. Besides, the overview part was included to portray the organization by its type, size, diversity of services for associating its software quality assurance practices. The questionnaires were arranged in major four sections which are discussed in following subsections.

3.2.1 Characterization. This section discovered the characterization of the organization in terms of its type, service age, number of IT personnel in testing and coding in that organization. Furthermore, this section also explored whether the company had dedicated quality assurance team or individual quality assurance personnel.

3.2.2 Software Testing Methodologies. This section provided the basic purpose of testing in the organization, if it was using any testing methodology. It also included choices about the process maturity and/or quality model, quality assurance activities. Furthermore, this section explored the capability of the organization to conduct automation in testing software.

3.2.3 Testing Team and Tools. This section was dedicated to derive the information regarding test team organization and testing tools as it finds the vitality of quality assurance and testing for interviewee's organization. It also captured the teams'association in the organization. This section explored the tools and its types of testing that were used in the organizations.

3.2.4 SQAT Challenges, Training, Education and Career Prospect. The section was included to explore the thoughts of the IT personnel of SQAT challenges, future possibilities of software quality assurance, curriculum and training from the tertiary institutions. This section also segmented to understand the satisfaction of the interviewees in terms of mental and financial soundness. 


\subsection{Survey Method}

A Face-to-face interview [10] was used as survey methodology which encompassed open and closed-end questionnaire. The interviews were arranged at the workplace of the interviewee. Each interview required 35-45 minutes on an average. The questionnaire was sent to interviewee prior to the face to face session for convenience. Printed and verbal explanation notes were provided to all the interviewees during the interview session. Proper measures had taken to ensure the confidentiality and privacy of interviewees and the respective organization.

\subsection{Sample Selection}

This survey was intended at the people at the different departmental level. There was more than one department accountable for the development of release of the software. Firstly, we had prepared a draft questionnaire. Then, that made over a small group of five software industries experimentally with the help of alumni of AIUB. A small number of adjustments were made based on findings and experience from the trial survey. Finally, four types of participants were aimed to perform the survey in the following sequence: the top-level executives, the managers, team leads, members of the development team and members of the SQAT team. For the survey, random sampling method [4] was used to select population. A qualitative approach was used as the survey's aim was to investigate and understand the differences. For that reason, random sampling was chosen over other sampling methods. 100 organizations and individuals were invited through official emails to support and participate in the survey. However, 47 organizations and individuals responded to participate. The participation rate may appear low in numbers; however, the authors believe that the sample would be adequate to signify the entire targeted population.

\section{SURVEY RESULTS}

\subsection{Characterization}

The goal of this section was to identify the characteristics and diversities of Bangladeshi software firms in terms of its type, service age, the ratio of tester and developer and existence of the dedicated testing team. Questionnaires were tailored to accomplish these tasks. Firstly, the year of foundation of the firm was asked to each of the interviewees. Later, this information was used to determine the years in service of the firm. In figure 11, the result shows that more than $50 \%$ ( 25 out of 40 ) of the software firms are in the service for less than 10 years. On the other hand, only three organizations were observed with more than 20 years in service. This indicates the recent rapid growth in the ICT sector of Bangladesh. However, the absence of software companies with more experience can be considered as an alarm for sustainable development in this sector. Secondly, the type of the organization was asked. This was a multiple-choice question because an organization can fall into different types based on its provided services.

Figure 2 depicts that $58 \%$ software development organizations are either product or service-based company. $24 \%$ organizations are considered themselves as outsource or offshore based firms. The percentage of the startup companies can be very pleasing from the authors'point of view. Because compared to the previous study [5], the percentage of startup has been almost doubled in past 2 years. Next, the interviewees were asked whether their organization have dedicated test team or not. Out of 47 respondents, 37 have dedicated test team in their organizations. However, in 10 organizations, they do not have any dedicated test team. Among these 10, 6 organiza-

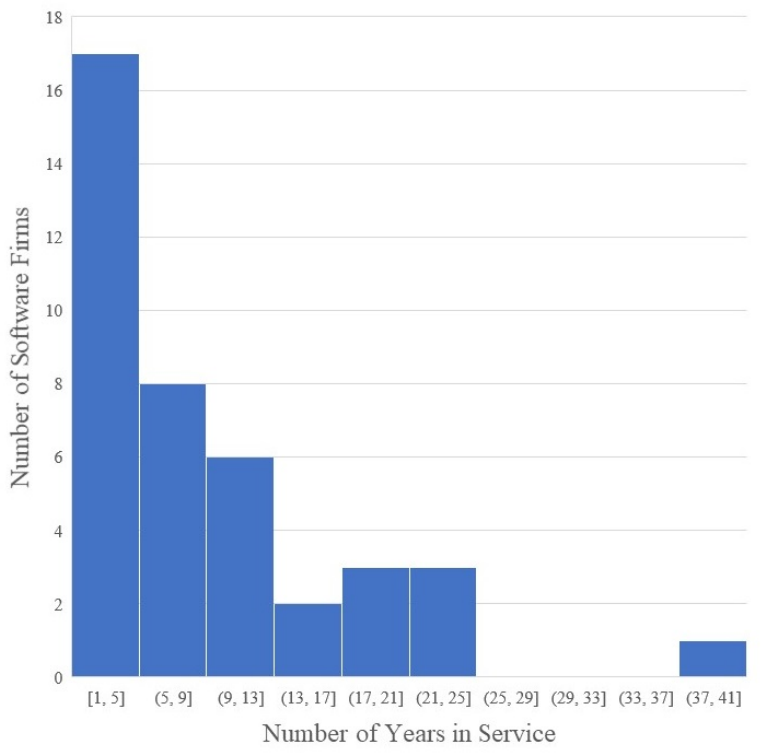

Fig. 1. Number of Years in Service by Software Firms.

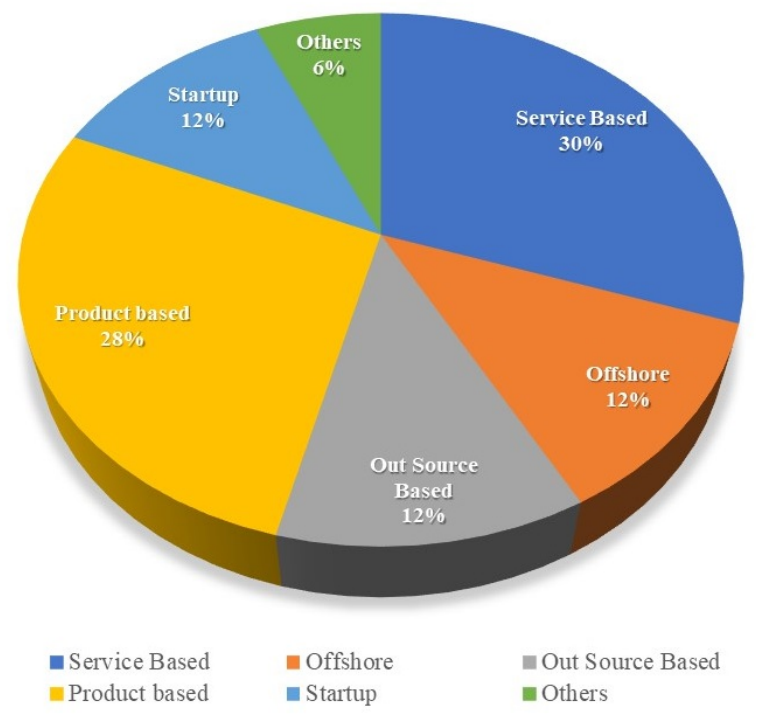

Fig. 2. Bangladeshi Software Firms by Service Types.

tions are in service for less than 5 years. To conclude the section, questions were asked to understand the ratio of testers and developers in software development organizations. Figure 3 shows the number of testers and developers in different organizations. From the figure it can be seen that, the number of organizations with the fewer tester is much higher than the number of organizations with fewer developers. In contrary, the number of firms with many developers are much higher than the number of firms with many testers. Further findings and recommendations are discussed in section V and VI. 


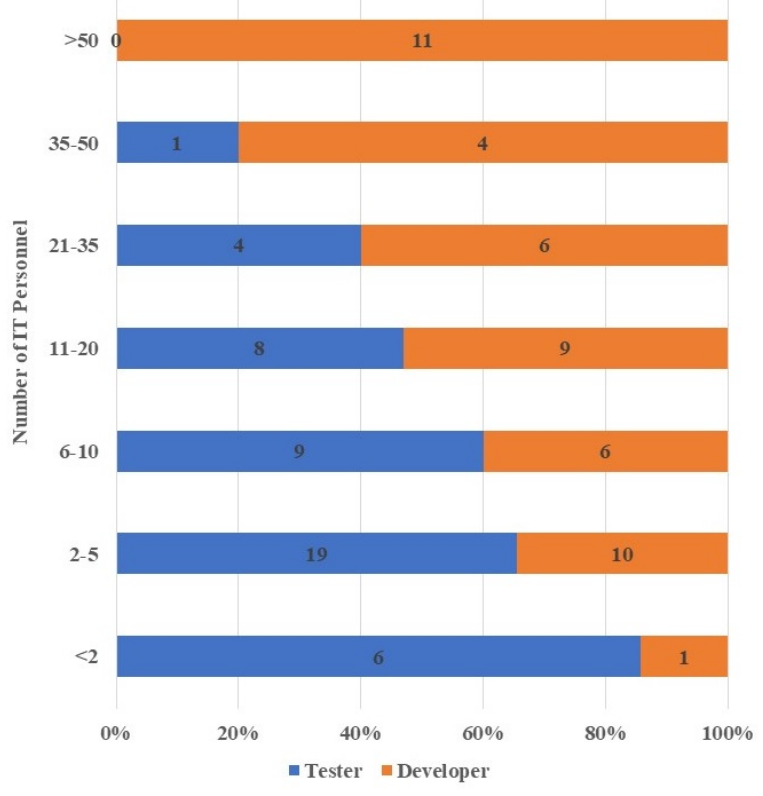

Fig. 3. Number of IT Personnel in Organizations by Tester and Developer.

\subsection{Software Testing Methodologies}

This section was aimed at identifying the SQAT practices among the software firms in Bangladesh. To achieve this goal, questions were asked to capture the main reasons behind performing testing, the usage of structured testing methodologies, the process maturity or quality models that are followed and other aspects of testing activities as well as effort allocation. At first, a question was asked to understand what is the main driving force for performing testing. From figure 4 we can see that almost 50\% respondents perform testing to ensure the quality of their software which is a good indication of the mentality among the interviewees. However, 22\% respondents do testing as it is their client's requirement. This indicates they are not so concerned regarding their software's quality which is not a good indication for the overall prospect of an organization.

Next question was related to following any structured testing methodologies. According to $68 \%$ respondents, they follow structured testing methodologies all-time. However, 9\% interviewees do not follow any structured methodologies at all and rest of the respondents follow sometimes. Then, the respondents answered the multiple-choice question regarding mostly performed QA activities. Figure 5 shows that testing is the most commonly performed QA activity followed by Inspection/Formal Review and Walkthrough/Informal Review.

Furthermore, we were also interested to know which testing activity receives most of the effort among different testing like functional, regression, aesthetics. Figure 6 illustrates that functional testing receives the most effort according to 20 respondents followed by Regression testing according to 12 respondents. However, we can observe that, automated testing receives very less effort. Yet, regarding the importance of automated testing, everyone agrees that it is an important part of the SQAT process. Besides, out of 47 respondents, only 24 respondents have performed automated testing on more than 5 The intention of the last question of this section was to distinguish the practices of process maturity

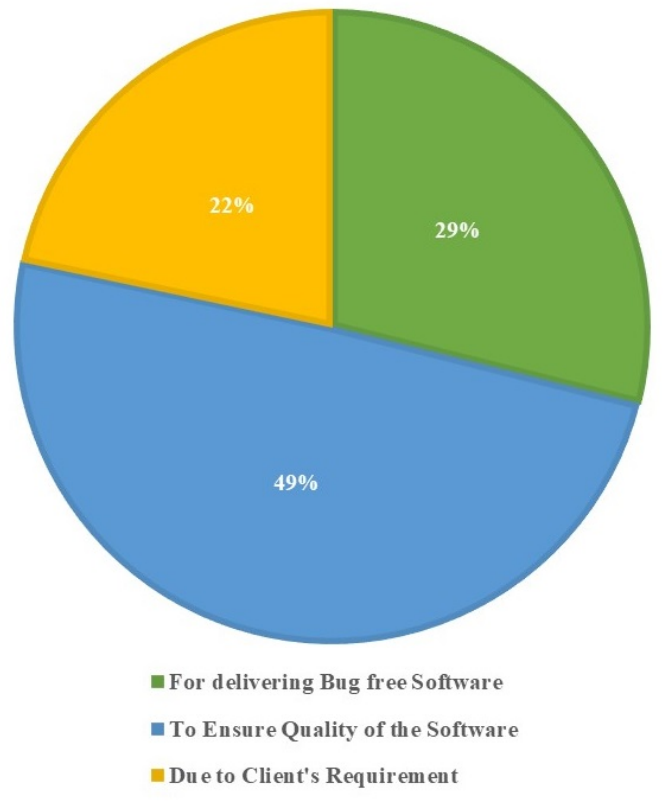

Fig. 4. Reasons behind Performing Testing.

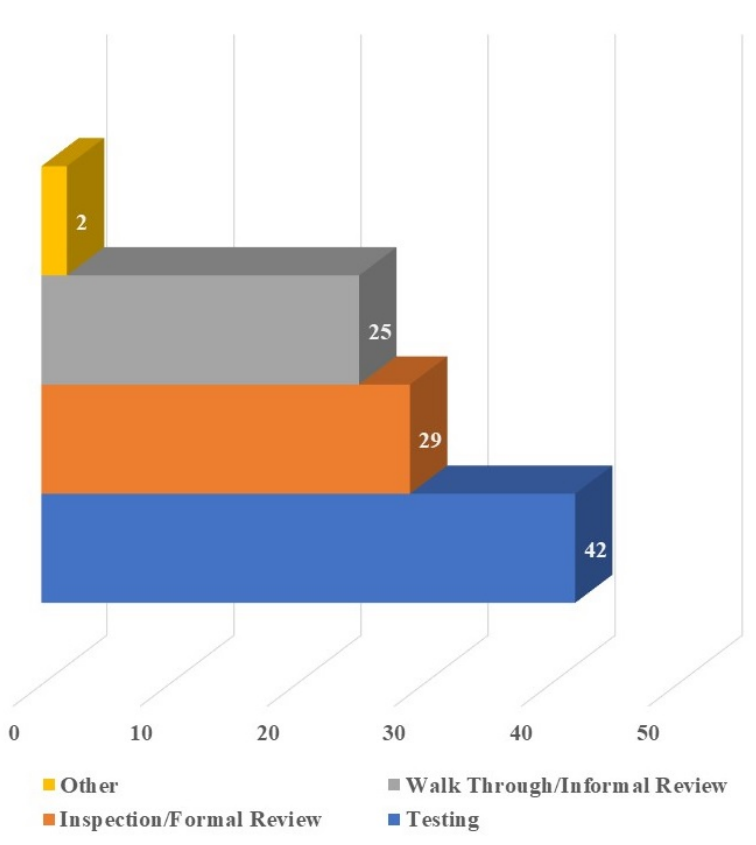

Fig. 5. Most Commonly Performed QA Activities.

and/or quality models among the Bangladeshi practitioners. A process maturity and/or quality model helps an organization to evaluate and improve its software development process. According to the responses, around 13\% software firms do not follow any kind of process maturity and/or quality model. Figure 7 illustrates the responses further. For further investigation regarding this issue, we looked at the average years in service of the software firms. Our assumption was that, new organizations might not be following any 


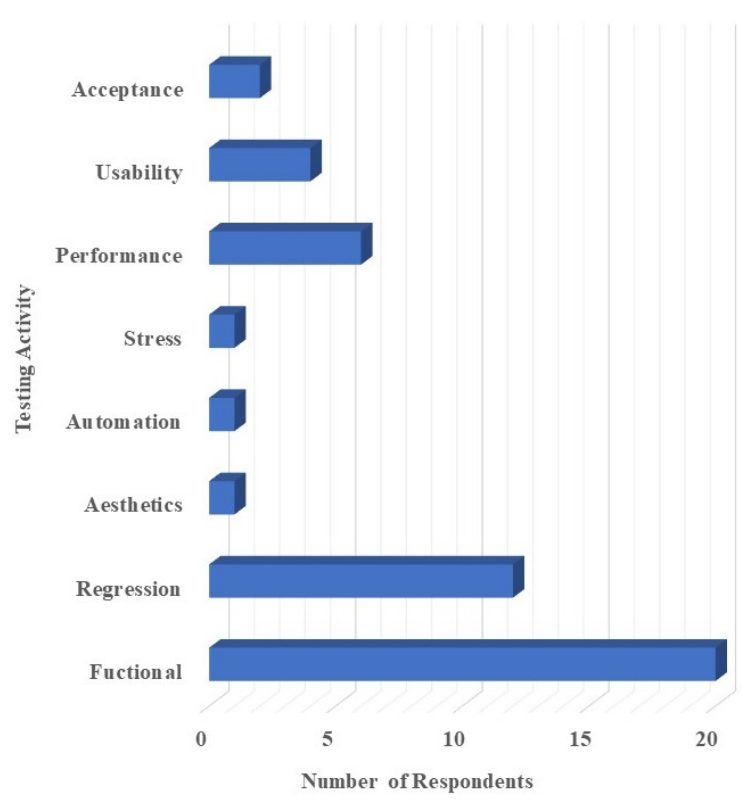

Fig. 6. Effort Received by Testing Activities.

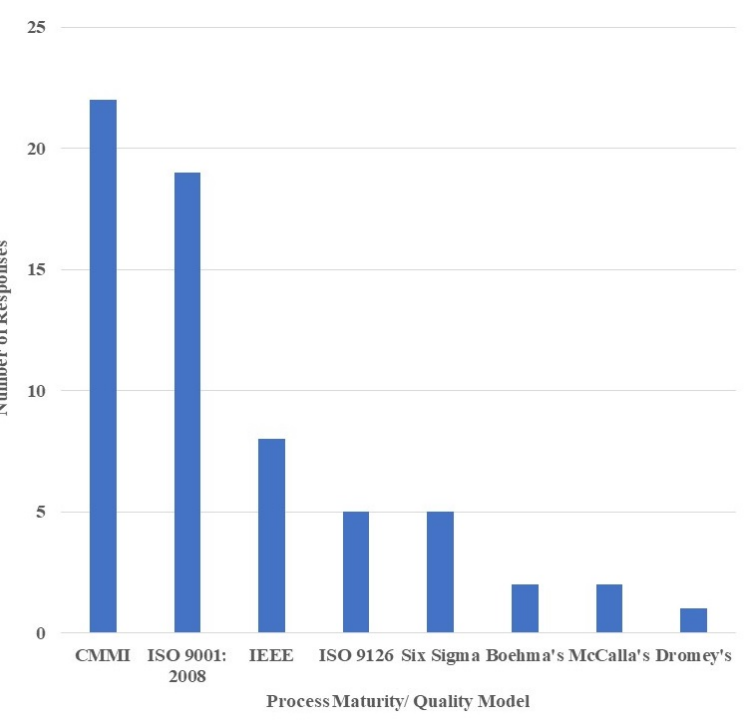

Fig. 7. Responses to Process Maturity/Quality Model.

maturity/quality model as they are new in the business. However, shockingly we have observed that the firms who are not following any maturity or quality model have the average age of 6.5 years in service. Further discussion regarding this issue is discussed in section V.

\subsection{Characterization}

The objective of this section was to capture the essence of SQAT, i.e. the testing team. Different aspects of the testing team, for instance, the structure of the testing team, the importance of testing team, conflict with the development team were investigated along with commonly used testing tools, types of commonly used testing

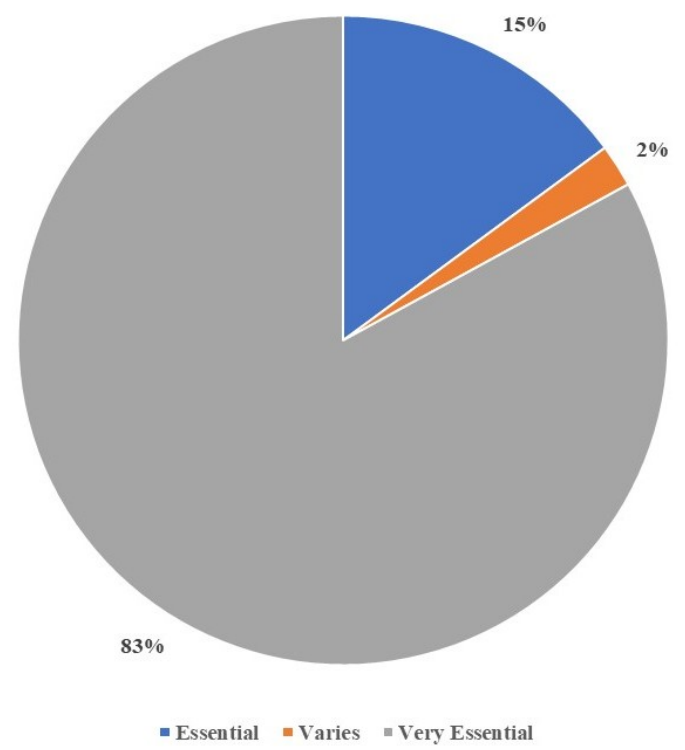

Fig. 8. Responses to Recognize the Importance of Testing Team in Organization

Table 1. Organization of Testing Teams in Bangladeshi Software Firms

\begin{tabular}{|l|l|l|}
\hline $\begin{array}{l}\text { Vertical Model (Project } \\
\text { Oriented) }\end{array}$ & $\begin{array}{l}\text { Horizontal Model } \\
\text { (Task Oriented) }\end{array}$ & Mixed Model \\
\hline 22 & 10 & 15 \\
\hline
\end{tabular}

tools and development of formal test plan. Firstly, we asked the interviewees how much important the testing team is for the success of their project from their perspective. Our initial assumption was the importance would be lower according to the responses from the development team side as they might tend to full stack development. However, figure 8 depicts that our initial assumption was wrong. $83 \%$ respondents think that testing team is essential to the success of the project. Among the 47 responses, no one says the testing team is not essential. Only $2 \%$ people think that the importance of testing team varies depending on the nature of the project. Yet, $21 \%$ (10 out of 47) interviewees do not have any dedicated test team in their organization or project.

The next question was designed for understanding the organization of testing team i.e., whether the testing team is project oriented or task oriented or a mix of both. The finding shows that vertical model i.e., a project orientated organization of testing team is the most commonly used test team organizing model among Bangladeshi software firms followed by a mixed model that combines both the vertical as well as the horizontal model.

Later, we studied whether the practice of developing formal test plan is common or not among Bangladeshi practitioners. 26 respondents perform developing formal test plan on a regular basis. On the other hand, 13 interviewees perform it sometimes. However, 8 respondents do not develop any formal test plan prior to testing. Furthermore, we also asked regarding the format of the formal test plan whose responses were positive to the previous question. 29 respondents follow their customized test plan outline. In contrast, 13 respondents follow the standard template for developing their 
Table 2. Frequency of Conflicts between Tester and Developer

\begin{tabular}{|l|l|}
\hline Conflict Frequency & Count of Responses \\
\hline Frequently & 20 \\
\hline Rare & 18 \\
\hline Very Frequently & 3 \\
\hline Very rare & 5 \\
\hline
\end{tabular}

Table 3. Responses to Commonly Used Testing Tools

\begin{tabular}{|l|l|l|}
\hline Name of the Tool & Type & No. of Responses \\
\hline JIRA & Issue Tracking & 27 \\
\hline Selenium & Functional Testing & 27 \\
\hline Apache JMeter & Performance Testing & 19 \\
\hline Appium & Mobile App Testing & 13 \\
\hline Redmine & Issue Tracking & 8 \\
\hline Helium & Web Testing & 5 \\
\hline Sikuli & GUI Testing & 5 \\
\hline TestComplete & Functional Testing & 3 \\
\hline Watir & Functional Testing & 2 \\
\hline Taurus & Performance Testing & 2 \\
\hline In-house built & Custom & 1 \\
\hline JUNIT & Unit Testing & 1 \\
\hline Ranorex & Functional Testing & 1 \\
\hline Gatling & Performance Testing & 1 \\
\hline TestLodge & Test Management & 1 \\
\hline Trello & Issue Tracking & 1 \\
\hline TestLink & Test Management & 1 \\
\hline Test Raid & Automated Testing & 1 \\
\hline BugZilla & Issue Tracking & 1 \\
\hline Postman & API Testing & 1 \\
\hline
\end{tabular}

formal test plan. This indicates the usage of the customized format of the formal test plan is very popular in Bangladeshi IT firms. This finding can provide new opportunities which are discussed in section 5 The next question was aimed at understanding the relation between test team and development team. So, we asked the interviewees how often do they face conflict with the development team. Table 2 summarizes the responses where we can see that, 20 respondents are facing conflict with the other team frequently. In contrary, 18 interviewees reply that they face rarely any conflict. Lastly, we explored the commonly used tools and tools'types related to performing testing in Bangladeshi IT sector. Table 3 summarizes the responses for commonly used testing tools where JIRA is the most popular issue tracking tool among the Bangladeshi software practitioners followed by Redmine. In case of web automation testing, Selenium is the most popular tool tailed by Helium. For mobile automation, Appium is the most common choice. However, we have observed that desktop automation is far behind compared to the web and mobile automation testing.

In case of the type of the testing tools, our hypothesis was that open source-based tools would be most common. The reason behind this hypothesis is that Bangladesh is a popular choice for out sourcing because of lower production cost. The commercial or Inhouse-built software will certainly increase the overall production cost. Figure 9 demonstrates the replies.

Figure 9 clearly supports our hypothesis as most of the respondents use open source-based testing tools followed by in-house-built.

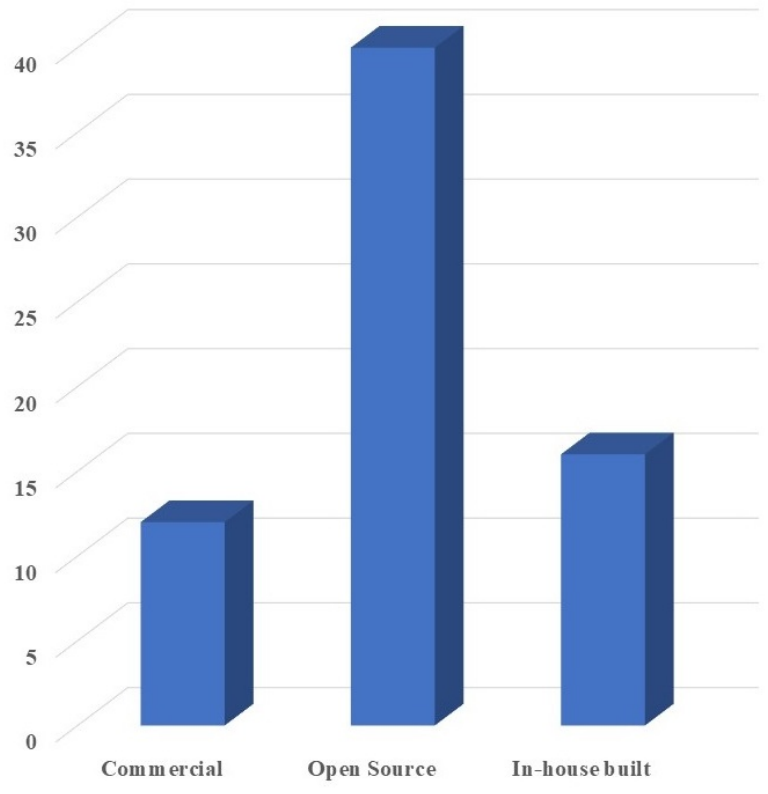

Fig. 9. Types of the Tools Related to SQAT.

\subsection{SQAT Challenges, Training, Education and Career Prospect}

The questionnaires of this section were tailored in such a way so that it can identify the challenges as well as training, education and career prospect of SQAT in Bangladesh. First, we asked the interviewees regarding the career prospect of the software testing in Bangladesh. According to $70 \%$ respondents, the career prospect as a tester is bright in Bangladesh. On the other hand, the career prospect is very bright according to $24 \%$ of the respondents. Next, we asked whether the curriculum of the educational as well as training institutes is adequate or not for a potential tester. $72 \%$ respondents think that the curriculum is not up to mark for a potential tester with different level of disagreeing and $79 \%$ interviewees strongly agree with adding more testing specific courses. In case of certification/ formal training of the SQAT personnel, we observed that $72 \%$ of the respondents do not have any kind of certification or formal training on testing. The subsequent question was intended to reveal the job satisfaction level of SQAT personnel in terms of job role and earnings along with other personnel. Figure 10 portrays that CEO/CTOs are the most disappointed personnel in terms of job role and salary in Bangladesh followed by SQAT personnel. In contrary, Managers are the most satisfied in Bangladeshi IT sector in terms of job role and earnings.

Lastly, we tried to identify the challenges of SQAT in Bangladesh by asking an open ended question. After analyzing the responses, we summarized the challenges as follows:

-Ensuring high quality software within given budget and time.

—Ensuring $100 \%$ test coverage.

- Thinking like an end-user for performing effective testing.

-Variations in terms of devices, networks, operating systems, demography of end users.

-Performing early testing.

—Understanding the product's requirements. 


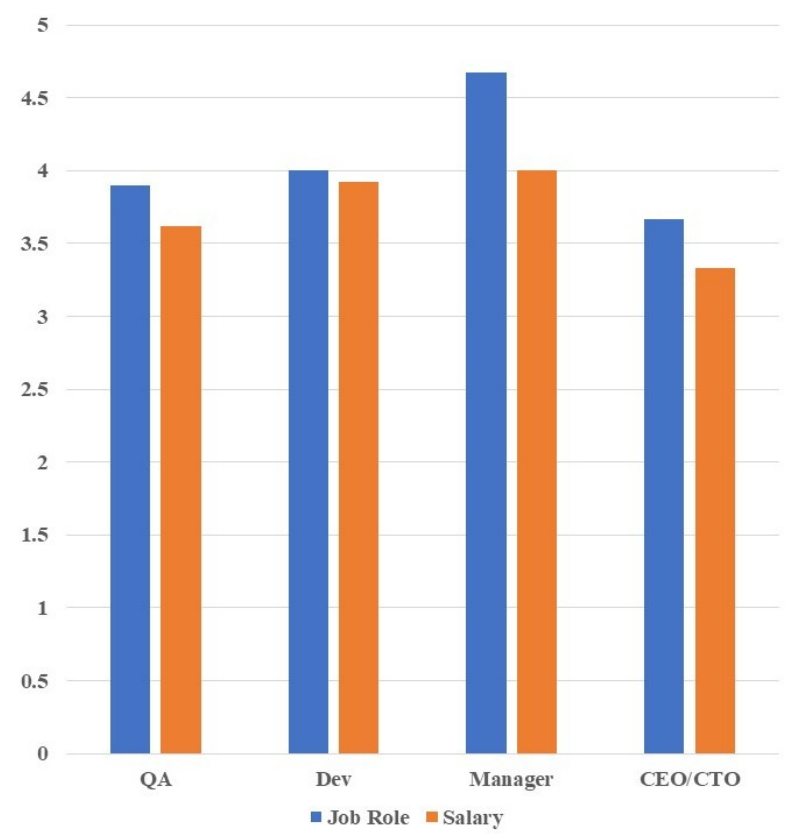

Fig. 10. Responses to Job Satisfaction of Different Personnel.

-Meeting Clients'expectations.

—Lack of Structured System for Testing and skilled Personnel.

-Performing testing activities while following Agile methodologies.

- Conducting performance testing.

\section{FINDINGS AND RECOMMENDATIONS}

This study provides plenty of insights regarding the software quality assurance and testing practices and challenges in Bangladeshi IT sector. This survey finds out that, the number of startups has been almost doubled in the past 2 years. This result clearly indicates the enthusiasm, skills, innovation of investors and IT personnel in Bangladesh as well as support from Government. Many of the software development organization do not have any dedicated test team. This finding may indicate their absence of maturity or financial capability to have dedicated test team. In this survey, we have observed that the number of developers is higher than the number of testing personnel. Furthermore, we have observed that in most the cases, the tester and developer ratio is 1:2. However, in some cases it is $1: 10$ which is obviously pretty stressful for a tester and overall for an organization to achieve its quality goal. The authors have also observed that some of the firms (22\%) perform testing as the clients'requirement. This outcome shows these organizations are not concerned regarding ensuring quality by themselves. Though we have observed that automated testing is considered as an integral part of the SQAT process according to all respondents, however, this testing receives less effort compared to other testing types. This may happen because not all projects require automated testing or not all organizations may have the required resources such as personnel, budget, tools to perform automation testing. Furthermore, this survey shows that automated testing has been conducted on a few percentages of projects. This can be an indication of unavailability of skilled automation test engineer or can be considered as the further scope in test automation sector. In case of process maturity or quality model, we have revealed that several software firms are not following any kind of process maturity and/or quality model. However, they are in the service sector for 6.5 years on average. It needs to be investigated further to identify the practices that are the reasons behind the success of the software firms who are not following any process maturity and/or quality model yet surviving in the competitive industry. This survey also indicates that few Bangladeshi IT firms achieved CMMI level 5. As 21 percent organizations do not have any dedicated test team, so this finding needs to be further investigated to identify the actual reason. This can be caused due to lack of budget, skilled personnel as most of them are either startup or outsource based company. The practice of developing formal test plan is not regular among Bangladeshi Practitioners. This indicates that further training and awareness programs are required to increase the practice of developing formal test plan among the practitioners. Furthermore, we have observed that many of the software firms use the customized format of the formal test plan. This creates the opportunity to develop the culture of sharing contents among the software developers. The conflict between the developer and SQAT personnel is a noticeable observation as $50 \%$ respondents say they are facing conflict on a regular basis. Necessary steps need to be taken to reduce this kind of trend although it is not a rare practice in software development. In case of test automation tools, we have observed that the usage of web and mobile testing automation tools is very common. However, in case of desktop testing automation tool, the situation is fully opposite. This indicates Bangladeshi software firms as well as training centers should focus more on desktop automation. Another finding of this survey is that most of the firms do not use commercial tools. This may be due to the fact that either the firms want to spend less or unavailability of test engineers expert in commercial tools. The findings of this study indicate the increasing demand of SQAT personnel for present and future in Bangladesh. Necessary steps need to be taken by the policy makers in the Government, training and educational institutes and software firms to fulfill this demand by delivering skilled SQAT personnel. As most of the interviewees express that the current curriculum in the educational institutes are not adequate, so these institutes should redevelop their curriculum so that they can nurture potential testers. Furthermore, this study reveals that most of the SQAT personnel do not have any certification or formal training which indicates the necessity of more training centers for SQAT training. This survey also reveals that testers are the least satisfied in terms of job role and salary compared to developers and managers. This finding suggests that, special attention needs to be paid to the prospect of SQAT personnel. Based on the findings from our survey, we recommend the following:

- Raise awareness among practitioners to make them understand the importance of SQAT for developing high-quality software through training, seminars and workshops. In this case, all the stakeholders i.e., Government, organizations, educational institutes, training centers should work in collaboration.

- The ratio of developer and tester should be reasonable. Organizations may practice different test effectiveness measures as well as performance measures to figure out the proper load of SQAT personnel.

- Process maturity and/or quality model should be practiced among the organizations. Further investigation should be undertaken to understand the reasons behind the organizations that are not practicing any process maturity and/or quality model. 
- To meet the demand of skilled SQAT personnel in the IT industry, the curriculum of the tertiary level educational institutes as well as training centers should be updated.

- The professional atmosphere should be created in the organizations to reduce conflict between tester and developer. They need to understand that they are not competitors to each other rather they are part of a team to deliver high quality software.

- Automated Testing should get more focus by the policy makers in the Government, IT Industry and educational institutes.

- To overcome the challenges in requirements analysis, educational institutes should include a course on requirements engineering and organizations should have specific personnel for requirements analysis (i.e. there should be a defined role for requirements analysis, like programmer, tester) in every software development project.

-Right now, there are only few training centers (e.g. New Horizons CLC, BITM-BASIS Institute of Technology \& Management) that offer training course on SQAT. More training centers should be established to produce more skilled test engineers.

\section{CONCLUSION AND FUTURE WORKS}

This paper presents the findings of the survey conducted to identify the software quality assurance and testing practices and challenges in Bangladesh. The authors along with the assistance of current students and alumni of American International University-Bangladesh (AIUB) steered the survey on software quality assurance and testing practices in Bangladesh between August, 2017 and January, 2018. A wide variety of IT firms, from CMMI Level 5 to newly established, from domestic to foreign-owned, were invited to participate in the survey. The authors believe that findings from this survey will help the policy makers in Government, Software firms and educational institutes to device their further action to nurture the SQAT sector in Bangladesh. As for future work, it is recommended to perform a more detailed survey of automated testing practices in Bangladesh. Also performing an SQAT survey only on startup companies would be interesting. In this study, we have observed that many of the respondents have identified requirement analysis as a challenge in SQAT. So as for future work it is recommended to conduct a survey to understand the current practices and challenges regarding Requirements Engineering in Bangladesh.

\section{REFERENCES}

[1] Exploiting potentials of the ict sector, Jun 2016.

[2] Shahid Al Noor, Golam Mustafa, Shaiful Alam Chowdhury, Md Zakir Hossain, and Fariha Tasmin Jaigirdar. A proposed architecture of cloud computing for education system in bangladesh and the impact on current education system. IJCSNS International Journal of Computer Science and Network Security, 10(10):7-13, 2010.

[3] AZM Ehtesham Chowdhury, Abhijit Bhowmik, Hasibul Hasan, and Md Shamsur Rahim. Analysis of the veracities of industry used software development life cycle methodologies. AJSE, 16(2):1-8.

[4] Floyd J Fowler Jr. Survey research methods. Sage publications, 2013.

[5] Vahid Garousi, Ahmet Coşkunçay, Aysu Betin-Can, and Onur Demirörs. A survey of software engineering practices in turkey. Journal of Systems and Software, 108:148-177, 2015.
[6] Vahid Garousi and Junji Zhi. A survey of software testing practices in canada. Journal of Systems and Software, 86(5):1354-1376, 2013

[7] David Gelperin and Bill Hetzel. The growth of software testing. Communications of the ACM, 31(6):687-695, 1988.

[8] Marie C Hoepfl. Choosing qualitative research: A primer for technology education researchers. 1997.

[9] Lena Karlsson, Åsa G Dahlstedt, Björn Regnell, Johan Natt och Dag, and Anne Persson. Requirements engineering challenges in market-driven software development-an interview study with practitioners. Information and Software technology, 49(6):588-604, 2007.

[10] Kate Kelley, Belinda Clark, Vivienne Brown, and John Sitzia. Good practice in the conduct and reporting of survey research. International Journal for quality in health care, 15(3):261266, 2003.

[11] SP Ng, Tafline Murnane, Karl Reed, D Grant, and TY Chen. A preliminary survey on software testing practices in australia. In Software Engineering Conference, 2004. Proceedings. 2004 Australian, pages 116-125. IEEE, 2004.

[12] Md Shamsur Rahim, AZM Ehtesham Chowdhury, and Shovra Das. Rize: A proposed requirements prioritization technique for agile development. In Humanitarian Technology Conference (R10-HTC), 2017 IEEE Region 10, pages 634-637. IEEE, 2017.

[13] Md Shamsur Rahim, AZM Ehtesham Chowdhury, Dip Nandi, and Mashiour Rahman. Issue starvation in software development: A case study on the redmine issue tracking system dataset. Journal of Telecommunication, Electronic and Computer Engineering (JTEC), 9(3-3):185-189, 2017.

[14] Md Shamsur Rahim and Ehtesham Chowdhury. Analyzing online voting systems for flaw detection. 2015.

[15] Md Shamsur Rahim, Md Hasibul Hasan, AZM Ehtesham Chowdhury, and Shovra Das. Software engineering practices and challenges in bangladesh: A preliminary survey. Journal of Telecommunication, Electronic and Computer Engineering (JTEC), 9(3-3):163-169, 2017.

[16] Md Shamsur Rahim, Md Hasibul Hasan, AZM Ehtesham Chowdhury, and Shovra Das. Software engineering practices and challenges in bangladesh: A preliminary survey. Journal of Telecommunication, Electronic and Computer Engineering (JTEC), 9(3-3):163-169, 2017.

[17] Colin Robson and Kieran McCartan. Real world research. John Wiley \& Sons, 2016.

[18] Gregory Tassey. The economic impacts of inadequate infrastructure for software testing. National Institute of Standards and Technology, RTI Project, 7007(011), 2002. 